\title{
Optimal Design of Low Pass Filter by Hamming, Hanning and Kaiser Technique
}

\author{
Akanksha Mahto', Muskan Chaubey ${ }^{2}$, Pranay Kumar Rahi ${ }^{3}$ \\ B.E. Scholar, Department of Electrical \& Electronics Engineering, Institute of Technology, Korba, C.G., India ${ }^{1,2}$ \\ Asst. Professor, Department of Electrical \& Electronics Engineering, Institute of Technology, Korba, C.G., India ${ }^{2}$
}

\begin{abstract}
In DSP application digital filter plays vital role. Therefore, digital filtering is one of the basic needs of digital signal processing. Digital filtering plays a significant role in the world of technology. Therefore, processing of input signal has to be done to get useful or wanted signal. This paper deals with the design of finite impulse response digital filter using window techniques the design based on MATLAB simulation. Through this paper the intricacies of the window methods are explained in a simple and a suitable manner. In this paper the comparative performance analysis of proposed filter are done with respect to Magnitude, Phase and Impulse. From simulation it is observed that Kaiser window shows better response in compare with other technique i.e. Hamming and Hanning.
\end{abstract}

Keywords: FIR Filter, DSP, Hamming Window, Hanning, Window, Kaiser Window, MATLAB, frequency sampling, amplitude-frequency.

\section{INTRODUCTION}

Digital filters play an important role in digital signal processing applications. They are widely used in digital signal processing applications, such as digital signal filtering, noise reduction, frequency analysis, multimedia compression, biomedical signal processing and image enhancement etc. A digital filter is a system which passes some desired signals more than others to reduce or enhance certain aspects of that signal. It can be used to pass the signals according to the specified frequency pass-band and reject the other frequency than the pass-band specification.

The basic filter types can be divided into four categories:

1. Low-pass

2. High-pass

3. Band-pass

4. Band-stop

1. TYPES OF DIGITL FILTERS-

1. Finite Impulse Response (FIR) filters

2. Infinite Impulse Response (IIR) filters[1]

Digital signal processing is the technique employed for the mathematical manipulation of an information signal so as to modify or improve it. For this purpose filters are mainly used. Digital filters are used in numerous applications e.g. control system, system for audio and video processing and communication system. Digital filters can be applied to very low frequency signals, such as those occurring in biomedical and seismic applications very efficiently. These are mainly two types of filter algorithms. They are finite impulse response filter (FIR) and infinite impulse response filter (IIR). In case of a FIR filter, the response due to an impulse input will decay with in a finite time. But for IIR filter, the impulse response never dies out. FIR filters are commonly known as non-recursive filters and IIR filters are known as recursive filters. The advantage of FIR filter over IIR filters are, FIR filter has linear phase and easily to control where as IIR filter has no particular phase and difficult to control. FIR filter is stable and depends only on input. FIR filters consists of only zeroes and IIR filters consists of both poles and zeroes [2].

\section{FIR FILTERS}

Finite impulse response (FIR) filter is a filter whose impulse response (or response to any finite length input) is of finite duration, because it settles to zero in finite time. A finite impulse response (FIR) filter is a filter structure that can be used to implement almost any sort of frequency response digitally. An FIR filter is usually implemented by using a series of delays, multipliers and adders to create the filter's output. FIR filters also known as feed forward or non recursive, or transversal filters. The unit impulse response is finite; so FIR filters are stable system 


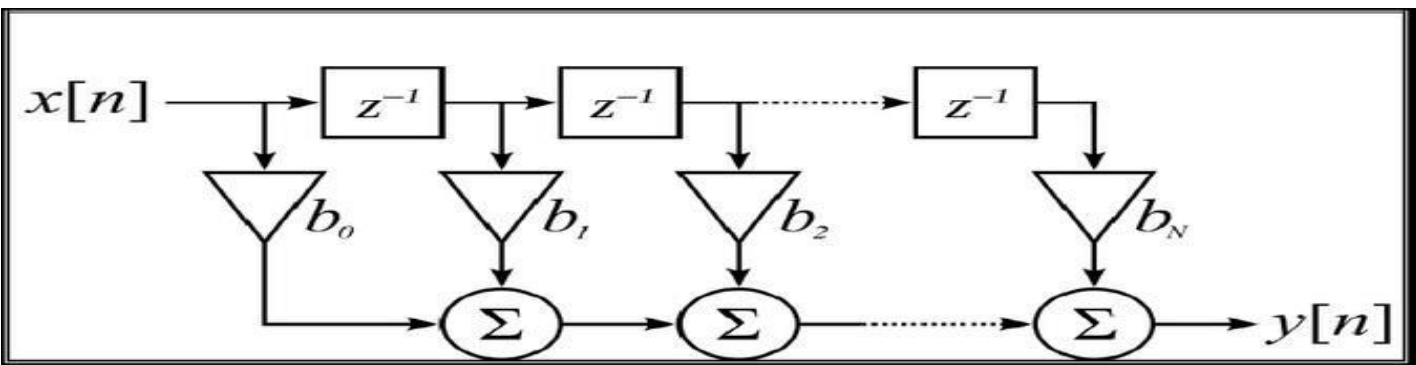

Fig. Block Diagram of FIR Filter

\section{IIR FILTERS}

IIR filters may be implemented as either analog or digital filters. In digital IIR filters, the output feedback is immediately apparent in the equations defining the output. Note that unlike FIR filters, in designing IIR filters it is necessary to carefully consider the "time zero" case in which the outputs of the filter have not yet been clearly defined.

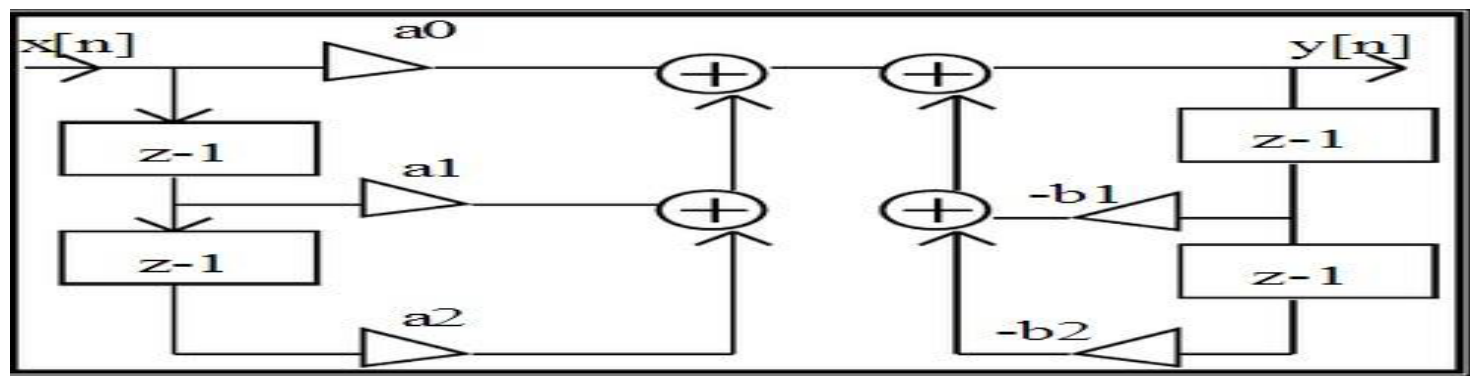

Fig. Block Diagram of IIR Filter

\section{FIR has following advantage over IIR Filter}

1. FIR filter is Finite IR filter and IIR filter is Infinite IR filter.

2. FIR filters are non-recursive. That is, there is no feedback involved. Where IIR filter is recursive. There is feedback involved

3. The impulse response of an FIR filter will eventually reach zero. The impulse response of an IIR filter may very well keep "ringing" ad-infinitum.

4. IIR filters may be designed to accurately simulate "classical" analog filter responses where as FIR filters, in general, cannot do this.

5. FIR filter has linear phase and easily control where as IIR filter has no particular phase and difficult to control

6. FIR filter is stable and IIR filter is unstable

7. FIR filter depend only on $\mathrm{I} / \mathrm{P}$ where as IIR filter depend upon both $\mathrm{I} / \mathrm{P}$ and $\mathrm{O} / \mathrm{p}$

8. FIR filter consist of only zeroes and IIR filter consist of both poles and zeroes.

\section{WINDOW FUNCTION-}

In this method, a certain bandwidth is generated using a truncated ideal low-pass filter, and then we use a selected window to get certain stop-band attenuation. In signal processing, a window function is a mathematical function that is zero-valued outside of some chosen interval. For instance, a function that is constant inside the interval and zero elsewhere is called a rectangular window, which describes the shape of its graphical representation. When another function or waveform/data-sequence is multiplied by a window function, the product is also zero-valued outside the interval: all that is left is the part where they overlap, the "view through the window" [4].

\section{WINDOWS AND THEIR EQUATIONS-}

Several window functions have been proposed. Listed below are some of the most common:

Rectangular window

Hanning window

Hamming window

Blackman window

Kaiser window

To reduce the oscillation in Fourrier series method, the fourier coefficient are modified by multiplying the infinite impulse response by a finite weighing sequence $(n)$ called a window. Windows are characterize by the main lobe width which is the bandwidth between first negative and first positive zero crossing, and by their ripple ratio. 
The main lobe far as possible in the main lobe and the energy in the side lobe should be as low as possible.

Two desirable characteristic of a window function are:

1)Fourier transform of the window function should have a small width of the main lobe.

2)Fourier transform of the window function should have side lobe that decrease in energy rapidly as tends to zero.

Window can be categorized as fixed or adjustable window function. Fixed window such as rectangular, hanning, hamming, and blackman window have only one independent parameter window length which control the main-lobe width. Adjustable window have two or more independent parameters such as window length and one more additional parameters that can control the other window's charactorstics. The Kaiser window is a kind of two parameter window. Function. In a Kaiser window width of main lobe can be controlled by adjusting the length of the filter and side lobe level can be controlled by varying the other parameter. But the Kaiser window has the disadvantage of higher computational complexity due to the use of vessel function in the calculation of the window coefficient.

However the amplitude of side lobe and ripples in pass band is also increased.

The function is

$$
(n)=\{\alpha-(1-\alpha) \cos (2 \pi n M-1), 0 \leq n \leq M-1\}
$$

Otherwise the value of $(n)$ is zero [5].

The windows used in this paper to design the FIR filter are:

Hanning window

Hamming window

Kaiser window

\section{HAMMING WINDOW TECHNIQUE}

Hamming window has a bell-like shape. Its first and last samples are not zero. The window is optimized to minimize the maximum side lobe. To eliminate the some pass band and stop band ripples, Hamming window technique is used. The coefficients of a Hamming window are computed from the equation.

$$
[n]=0.54-0.46 \cos (2 \pi) x \mathrm{nN}, 0 \leq \mathrm{n} \leq \mathrm{N} 2
$$

Richard W. Hamming observed that the sidelobes of the rectangular and Hanning windows phase reversed relative to each other, so a linear combination of the two would tend to cause them to cancel each other. He searched for the linear combination that minimized the maximum sidelobe amplitude and came up with the following formulation, which represents [6].

\section{KAISER WINDOW TECHNIQUE}

Kaiser window is very useful in practice. Zero-th order modified Bessel function of first kind

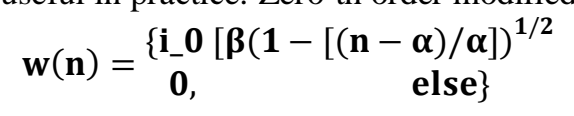

where $\mathrm{a}=\mathrm{M}=2, \mathrm{I}($ (.) is Bessel function, $\mathrm{b}$ is a parameter that determines to some extent the .shape. of the _lter. $\mathrm{M}$ and $\mathrm{b}$ trade off sidelobe amplitude and mainlobe width.

Question: which one obviously controls mainlobe width? $M$ and $\beta$ controls both width and tapering off (i.e., as b increases, width gets large but sidelobe amplitudes get smaller.)

Kaiser empirically found formula going from $\delta, w_{s}, w_{p}$ to $M$ and $b$.

$$
\begin{gathered}
\Delta \mathrm{w}=\mathrm{w}_{\mathrm{s}}-\mathrm{w}_{\mathrm{p}} \\
\mathrm{A}=-20 \log _{10} \delta
\end{gathered}
$$

This makes it very easy to do filter design very quickly .

Key: this gives the window w[n], which is to be multiplied by the ideal impulse response hd[n] to get the actual_lter

$$
\mathbf{h}[\mathbf{n}]=\mathbf{w}[\mathbf{n}] \mathbf{h d}[\mathbf{n}] \text {. }
$$

$\mathrm{M}$ does not change ripple error (to achieve d) this is determined by the sidelobe amplitude (determined by b, or type Of window).

So, better widths at same approximatin error =Kaiser window is probably the best so far [7].

\section{HANNING WINDOW TECHNIQUE}

The window function of a causal Hanning window is given by 


$$
\begin{gathered}
\mathbf{w}_{\text {Hann }}(n)=\left\{\begin{array}{c}
0.5-0.5 \cos \frac{2 \pi n}{N-1}, 0 \leq n \leq N-1 \\
0, \text { otherwise }
\end{array}\right\} \\
w_{H}(n)=w_{R}(n)\left[0.54+0.46 \cos \frac{2 \pi n}{N-1}\right]
\end{gathered}
$$

\section{DESIGN SIMULATION -}

To design the low pass FIR filter using MATLAB Parzon, Taylor and Hamming Window the parameter specifications are given in table 1 as

Table (1). Parameter specification

\begin{tabular}{|c|c|}
\hline PARAMETER & VALUE \\
\hline Sampling frequency(fs) & 48000 \\
\hline Cut off frequency (fc) & 10000 \\
\hline Order & 20 \\
\hline
\end{tabular}
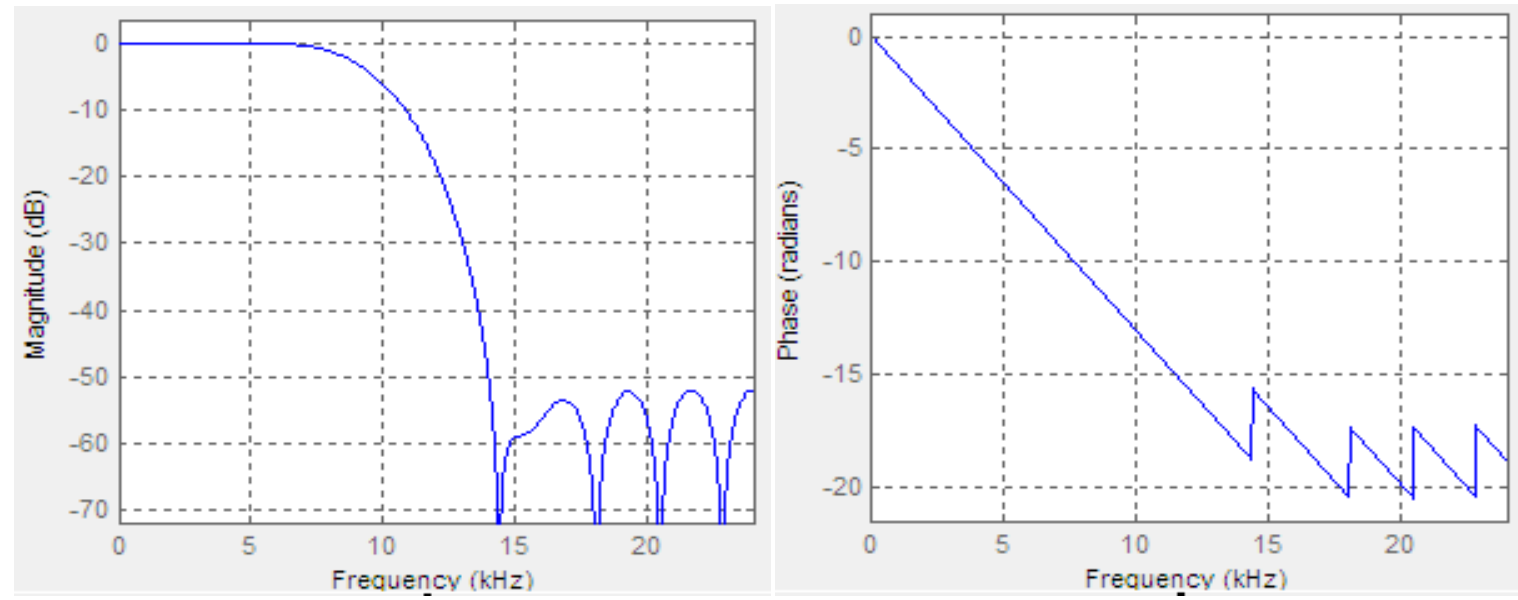

Magnitude and Phase response of Hamming Window Technique

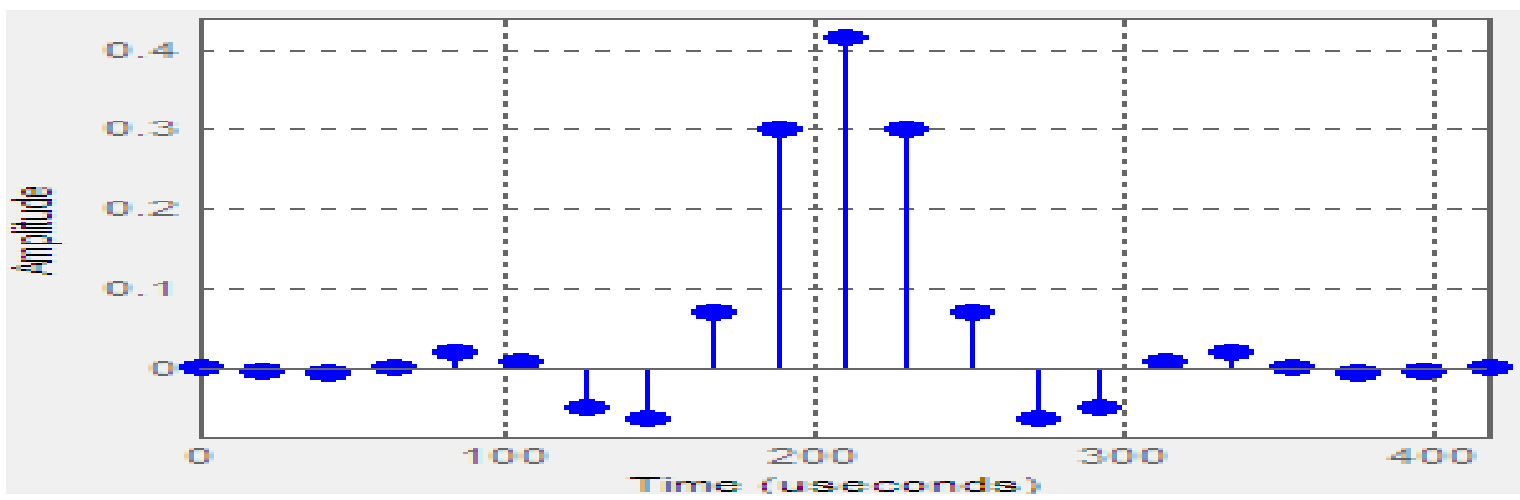

Impulse response of Hamming Window Technique

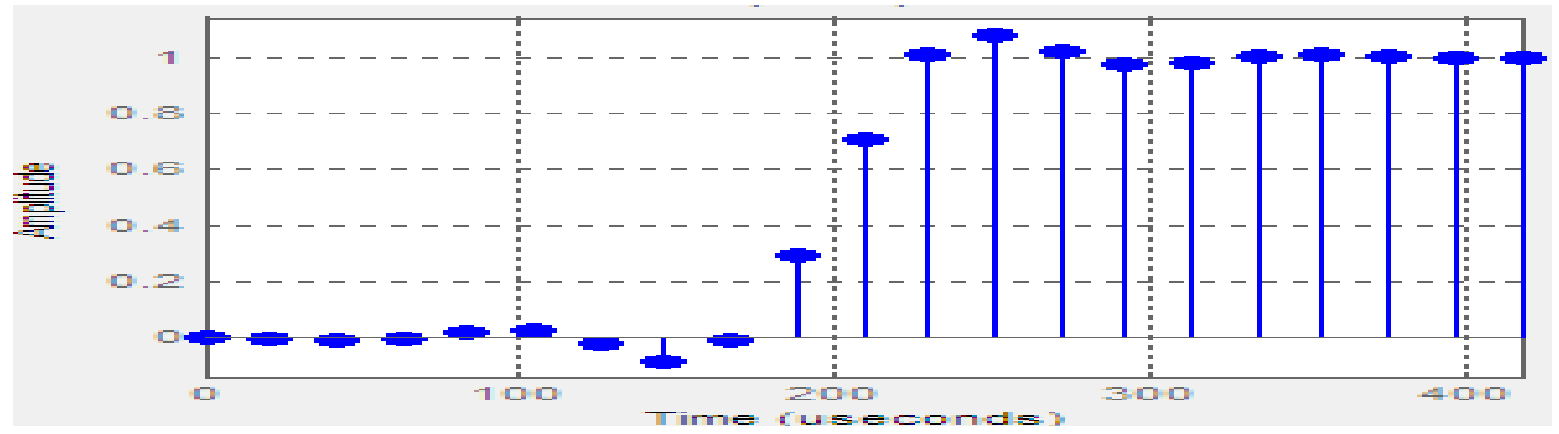

Step response of Hamming Window 


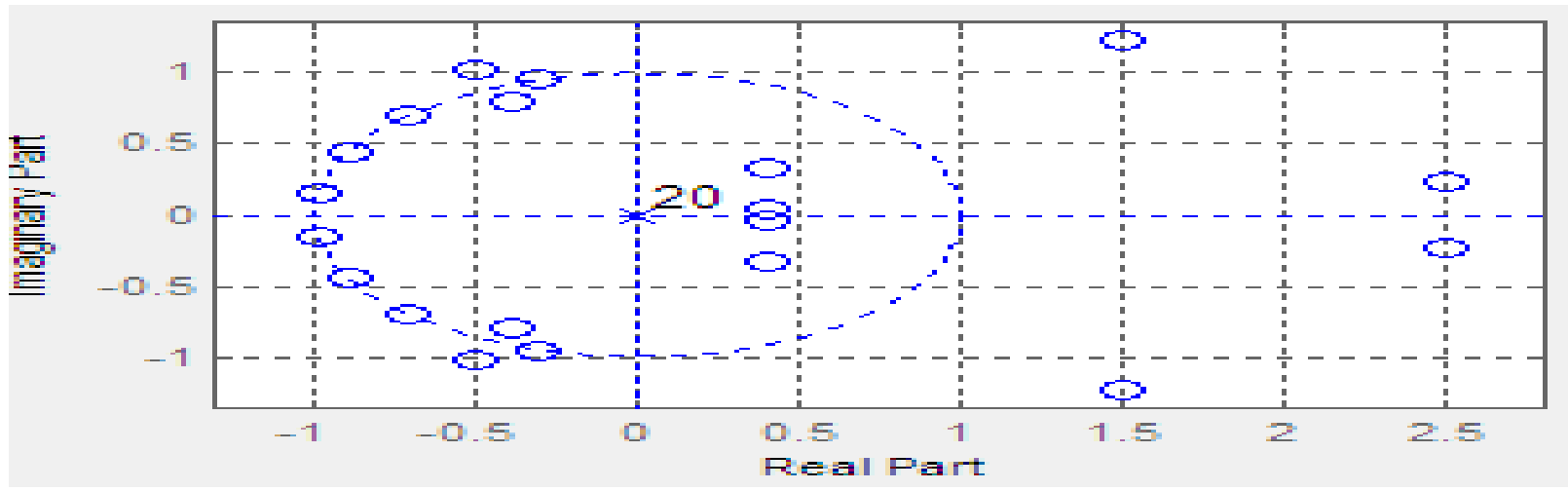

Poles and zeros plot of Hamming Window

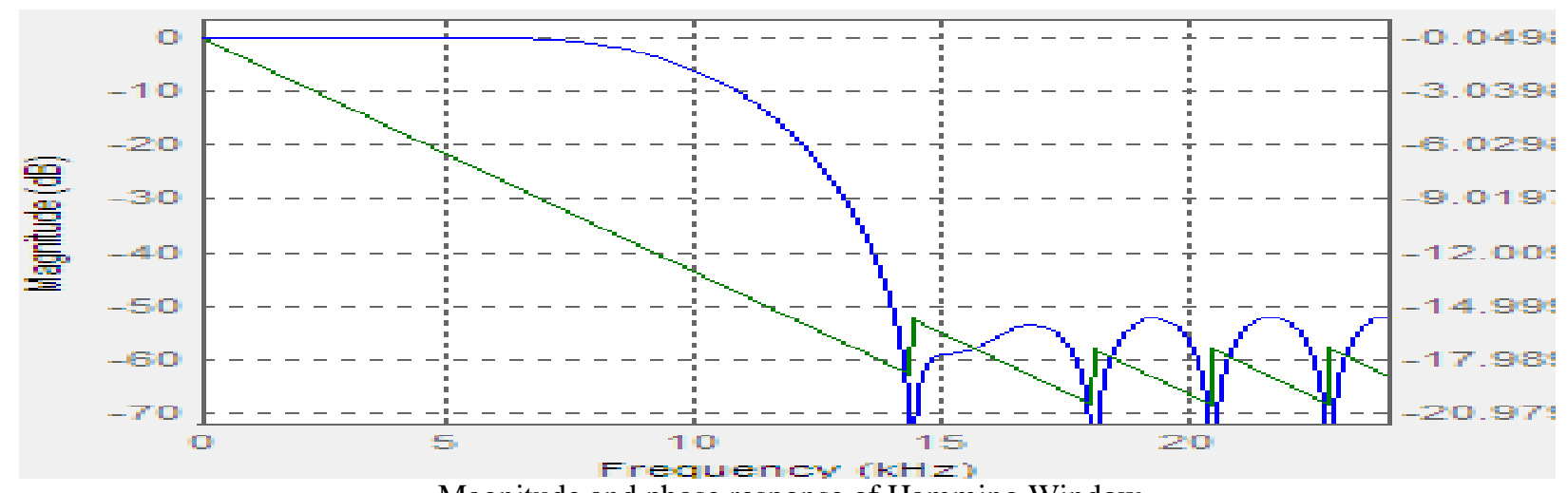

Magnitude and phase response of Hamming Window

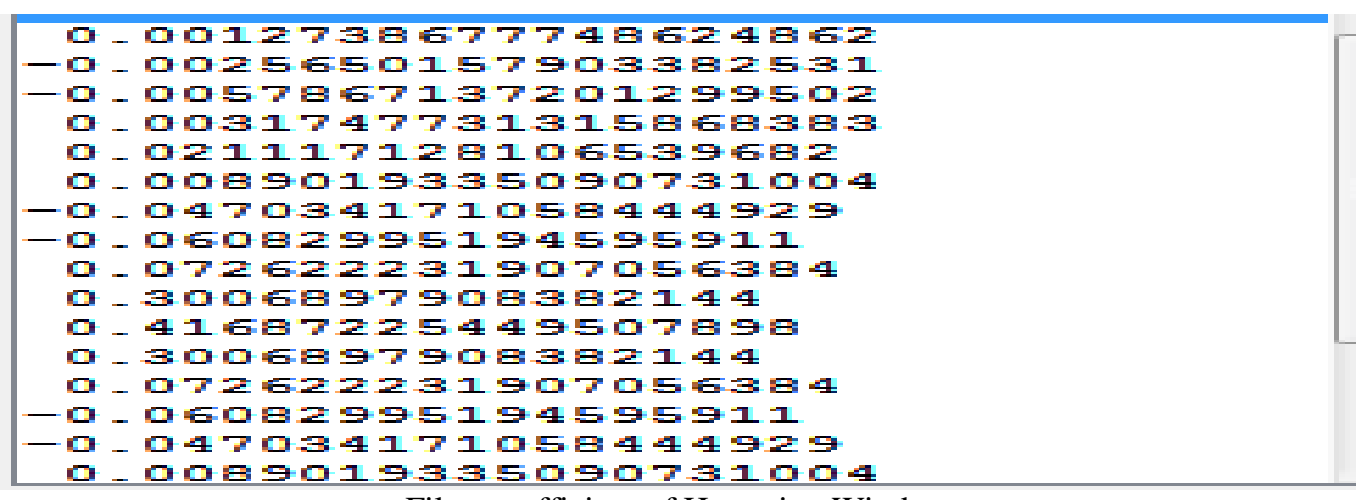

Filter coefficient of Hamming Window
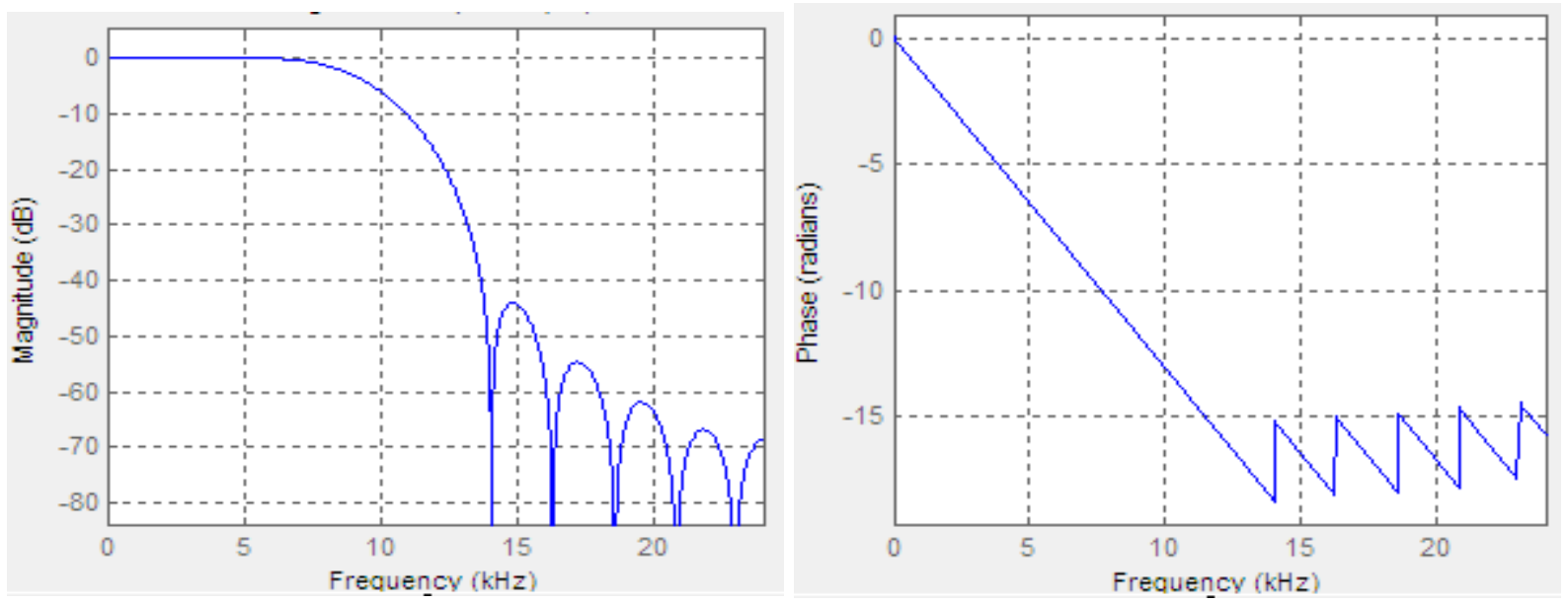

Magnitude and Phase response of Hanning Window Technique 

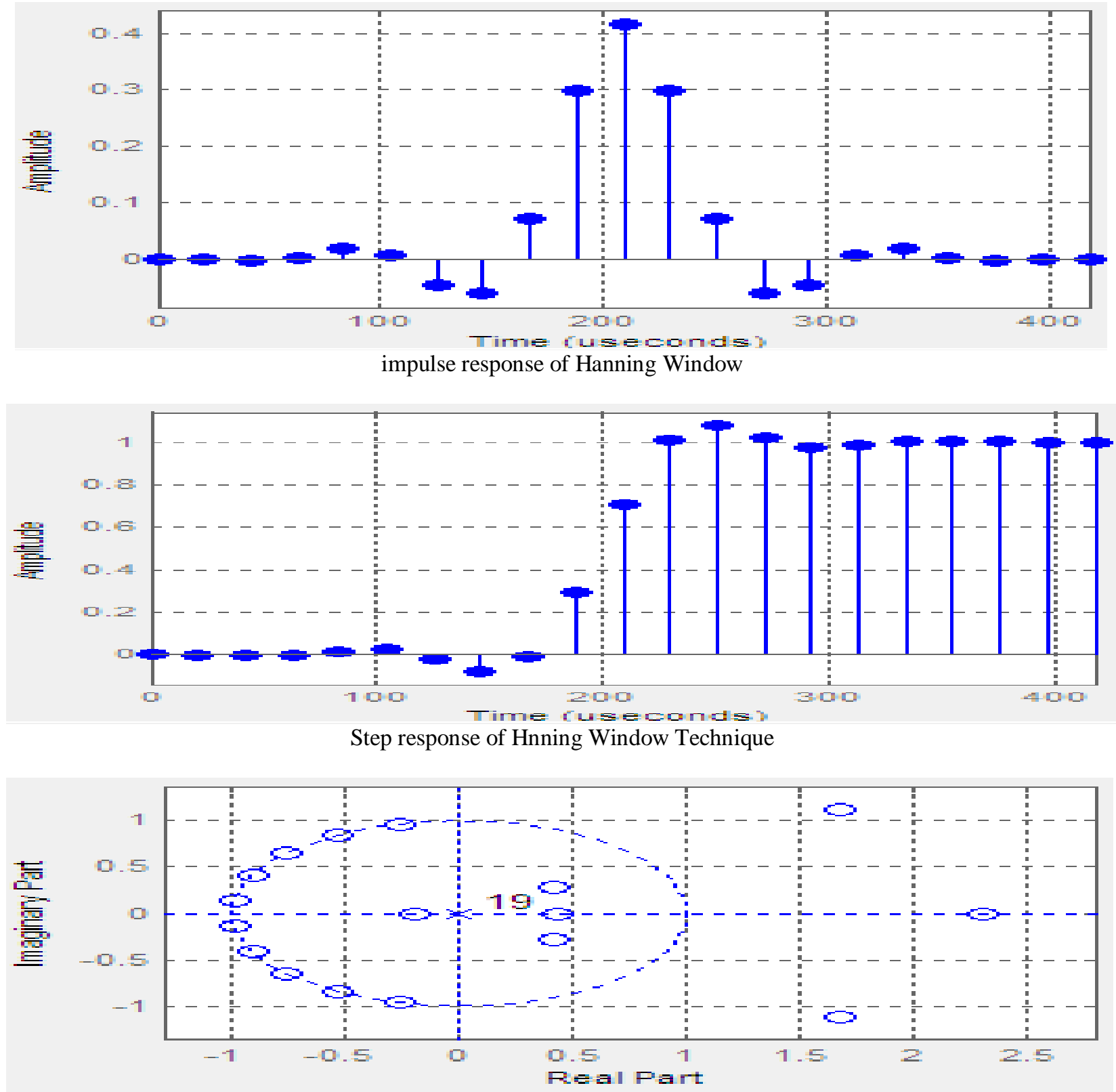

poles and zeros plot of Hannning Window

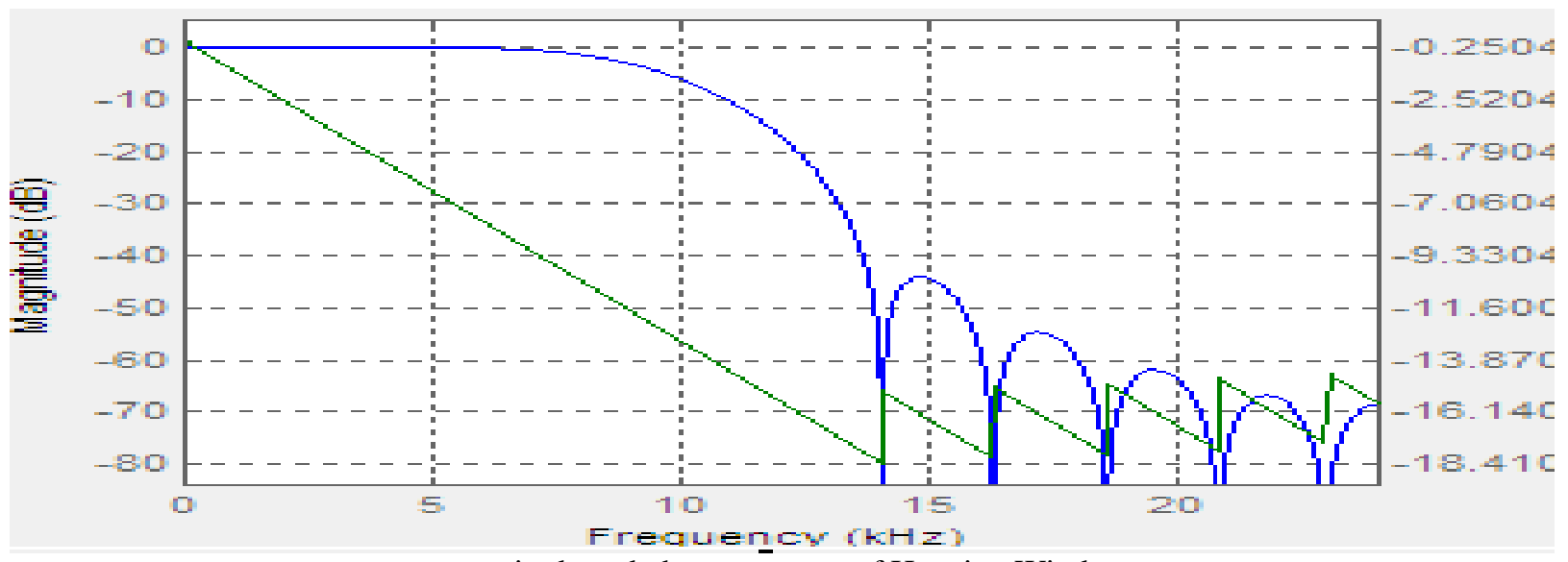

magnitude and phase response of Hanning Window 


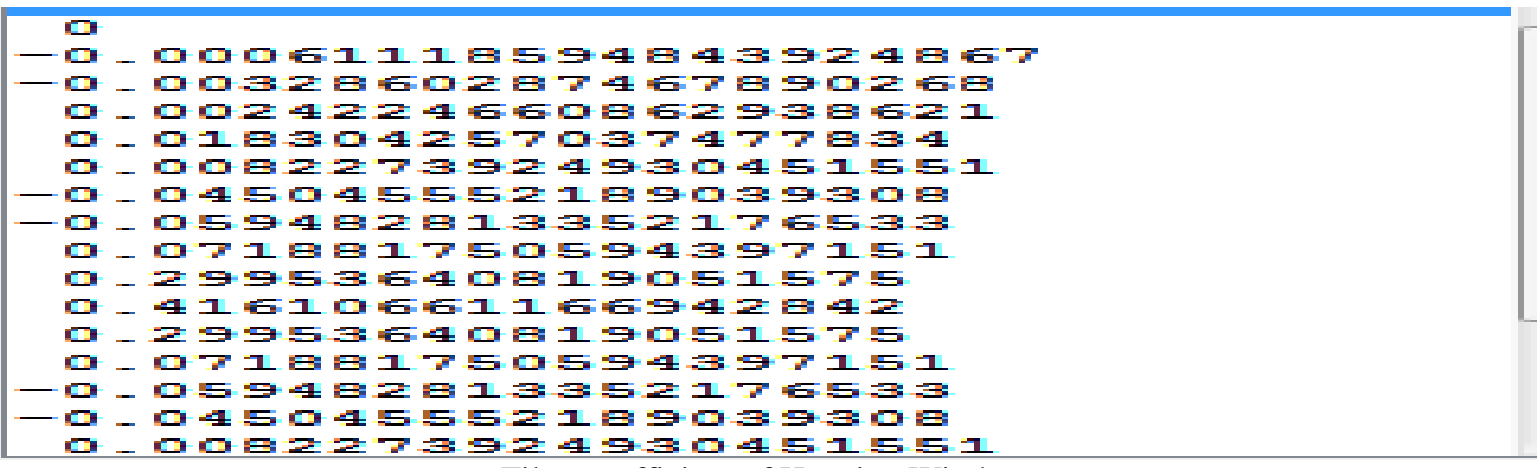

Filter coefficient of Hanning Window
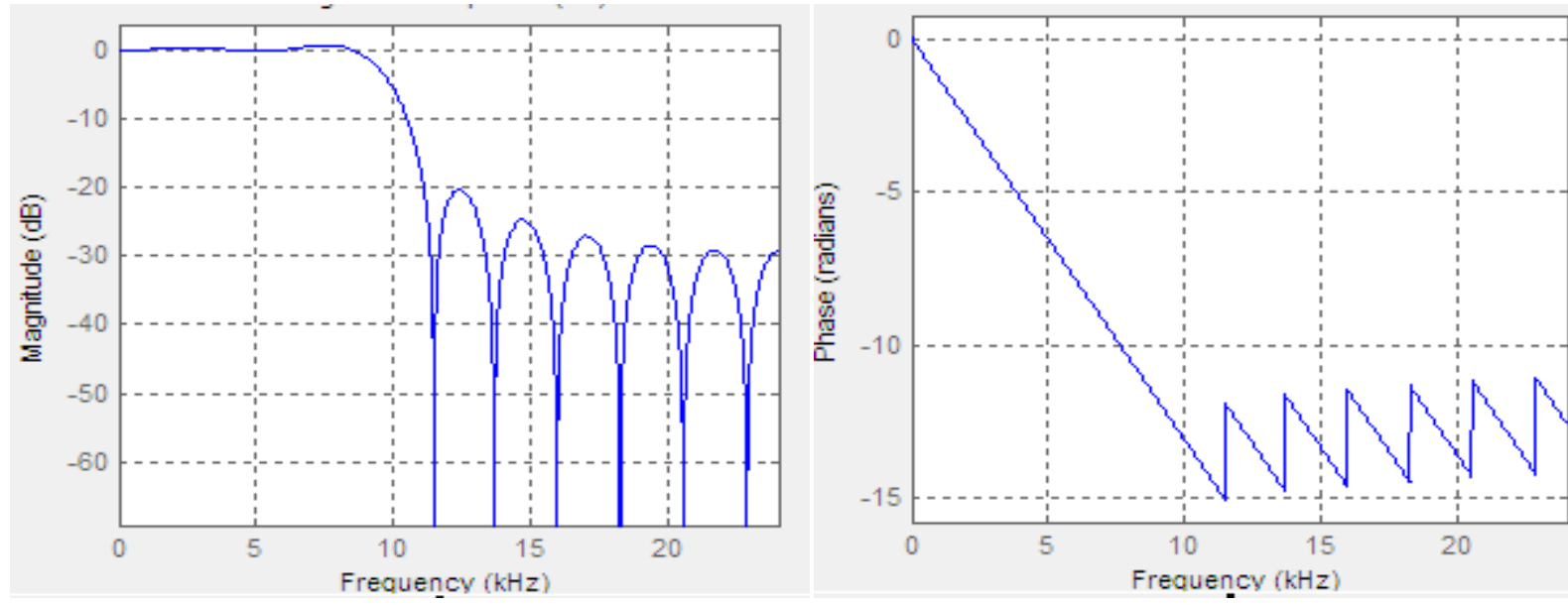

Magnitude and Phase response of Kaiser Window Technique

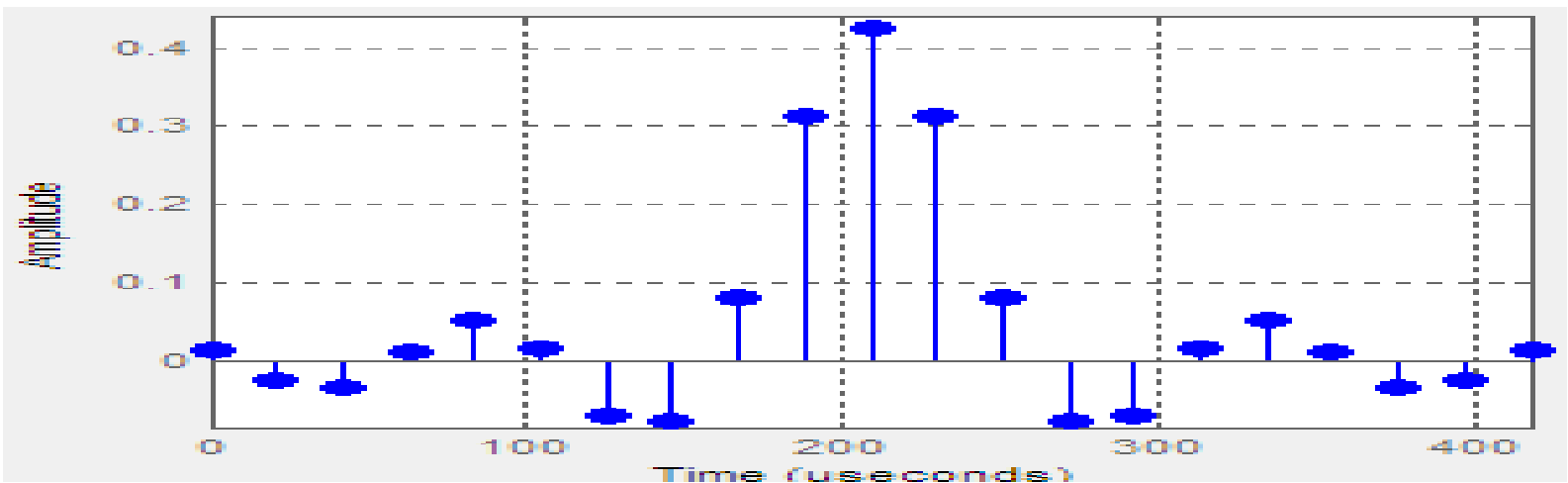

Impulse response of Kaiser Window

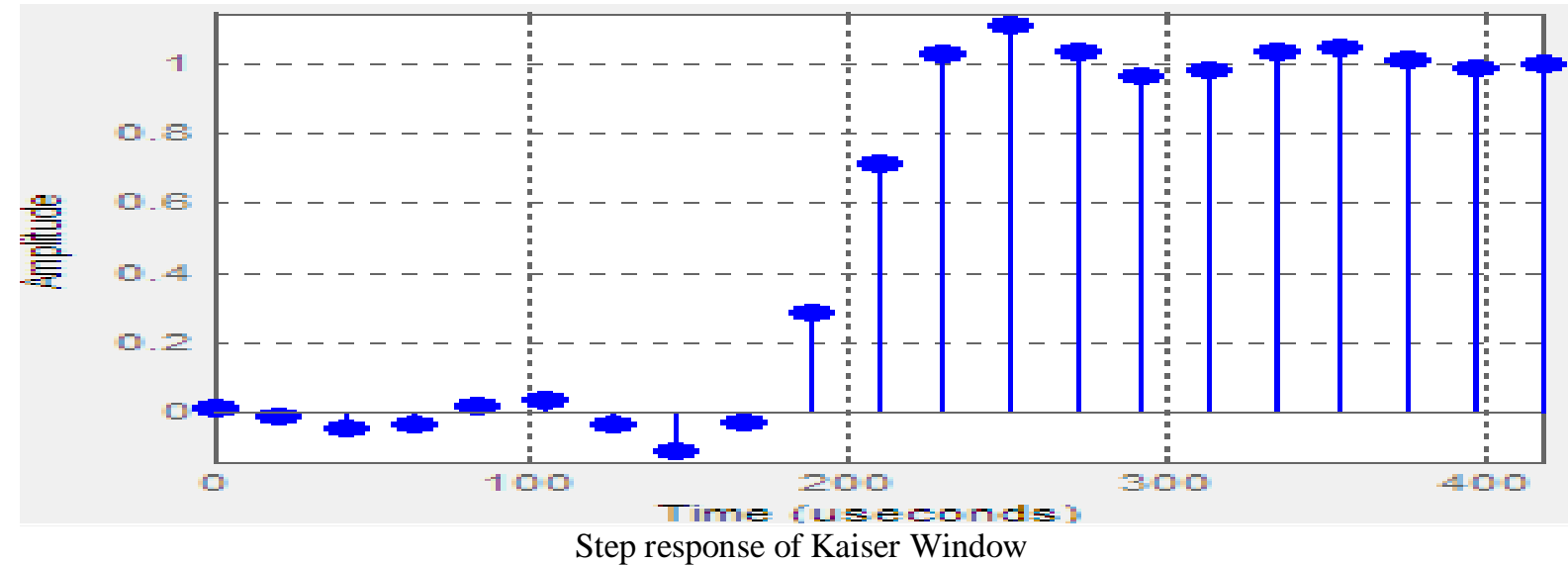




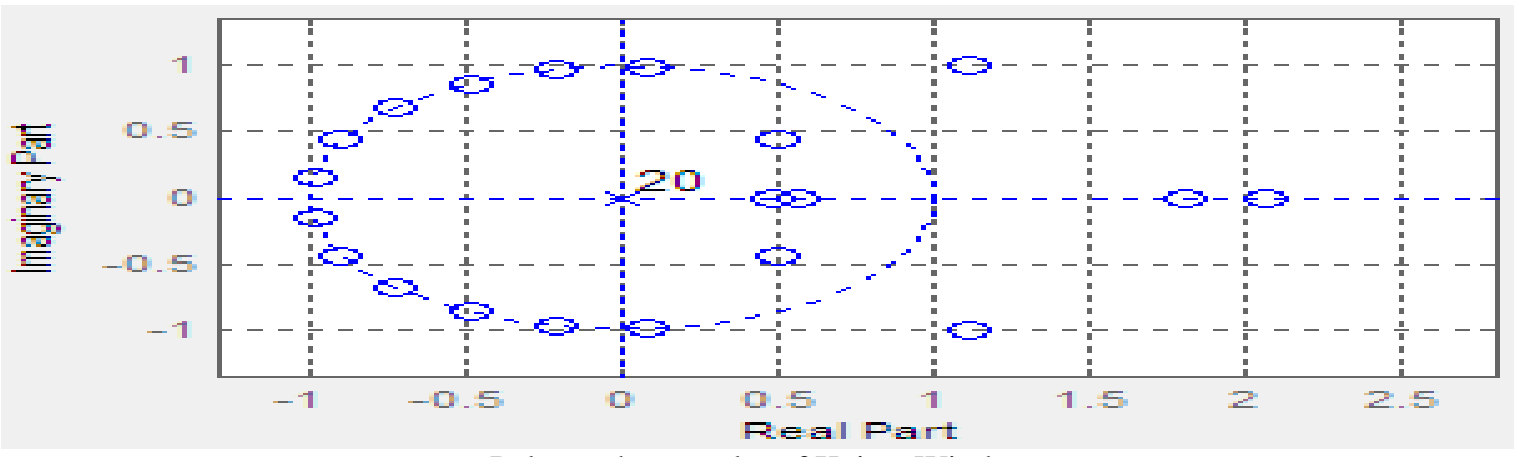

Poles and zeros plot of Kaiser Window

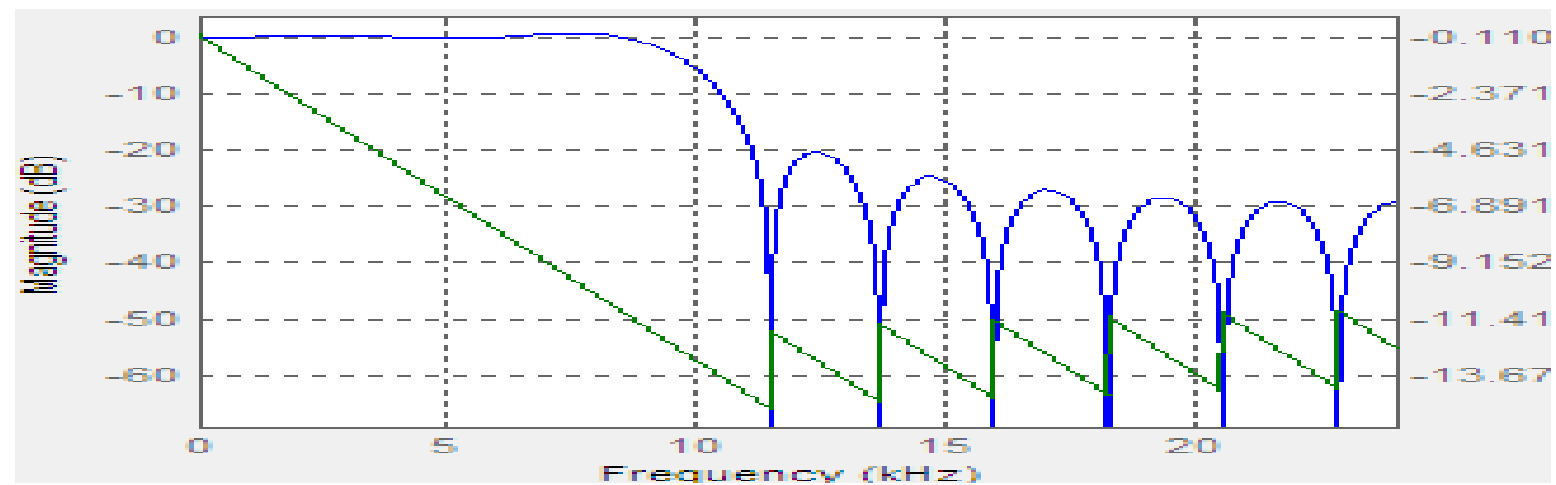

Magnitude and phase response of Kaiser Window

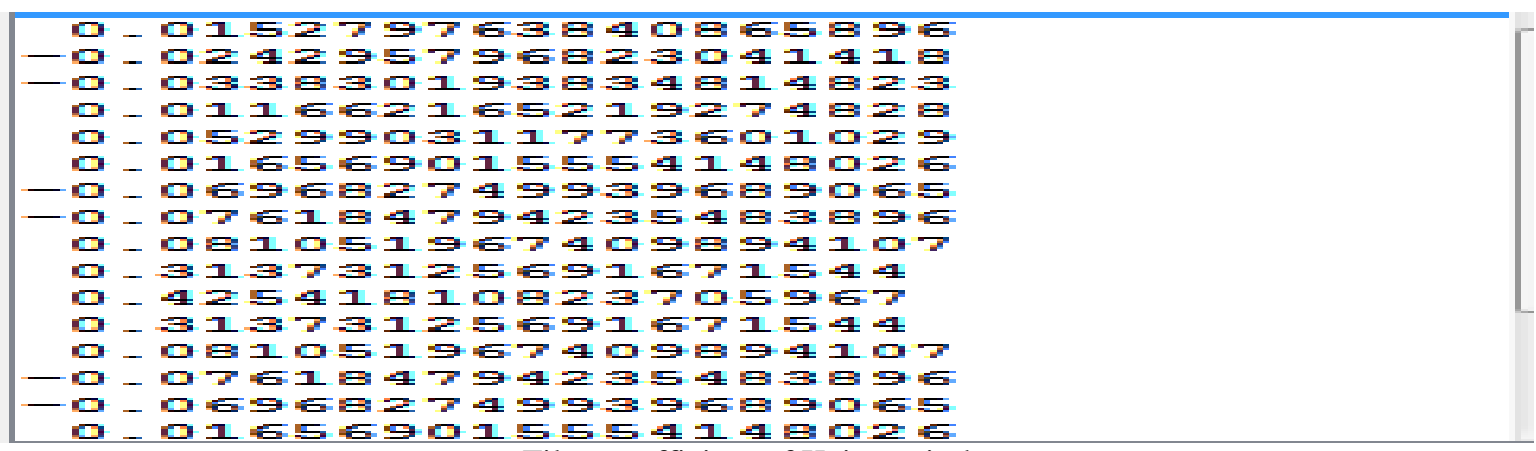

Filter coefficient of Kaiser window

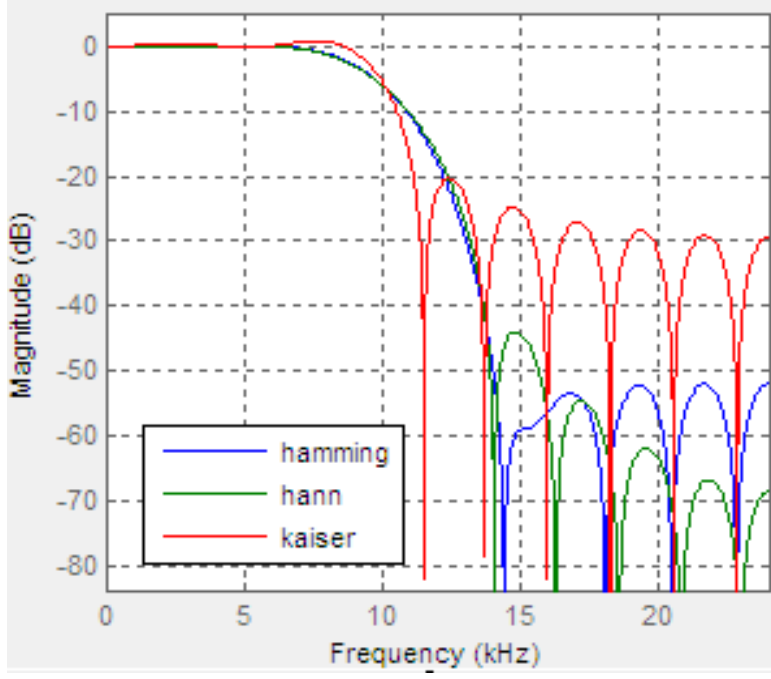

Magnitude comparison of Hamming, Hann and Kaiser

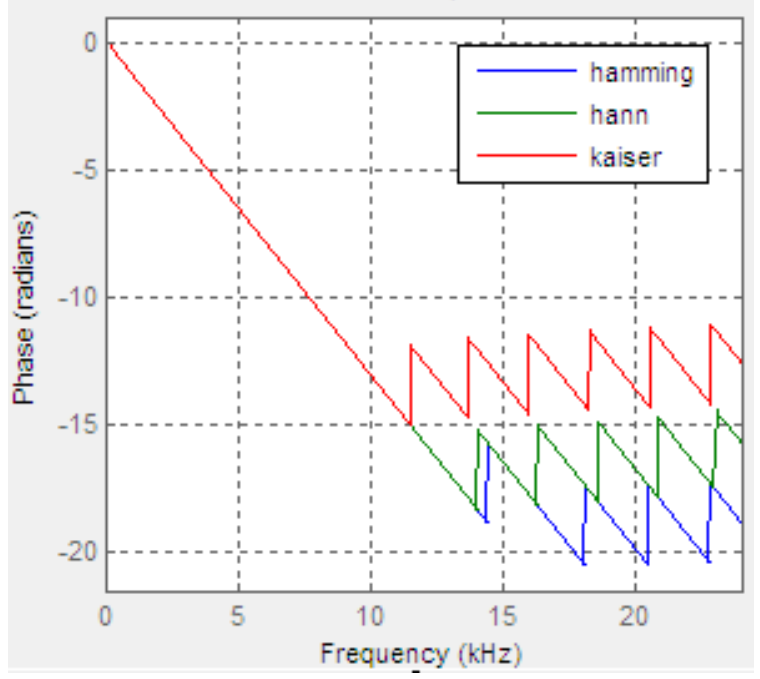

Phase comparison of Hamming, Hann and Kaiser 


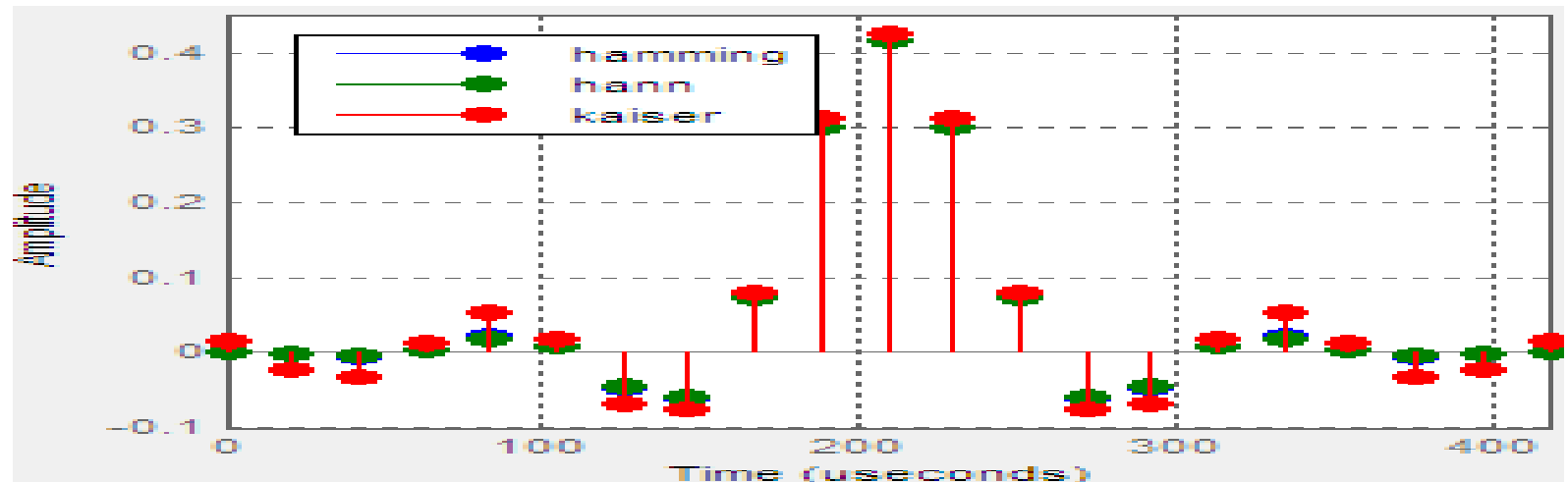

Impulse comparison of Hamming, Hanning and Kaiser

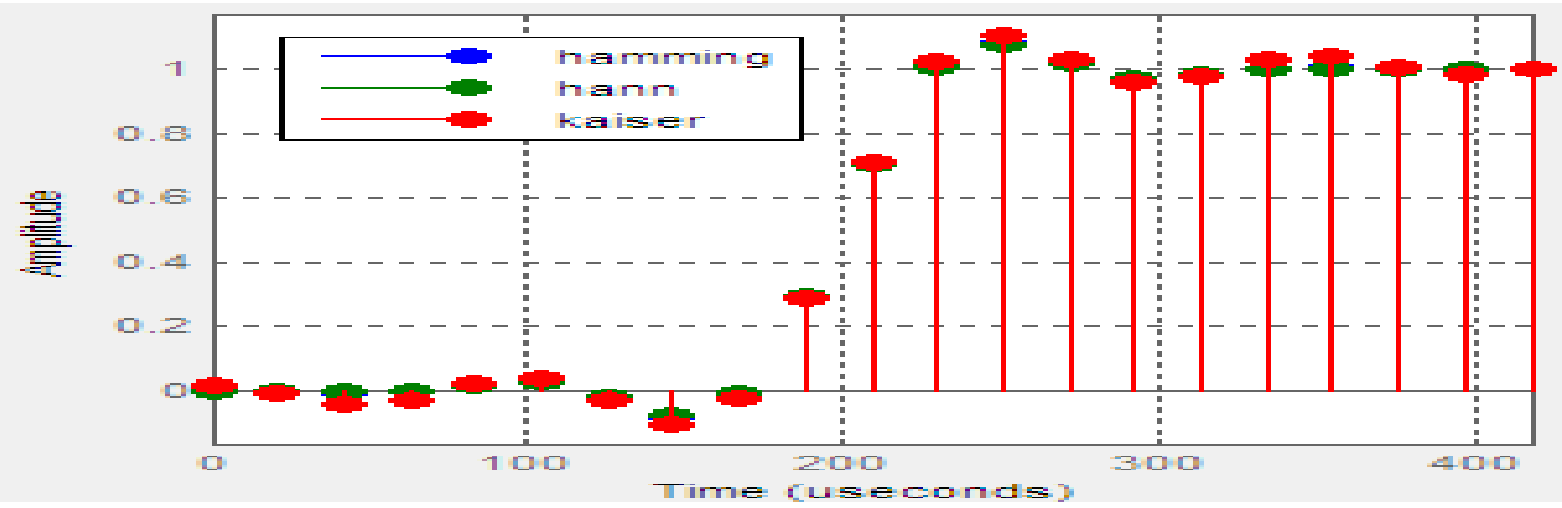

Step comparison of Hamming, Hanning and Kaiser

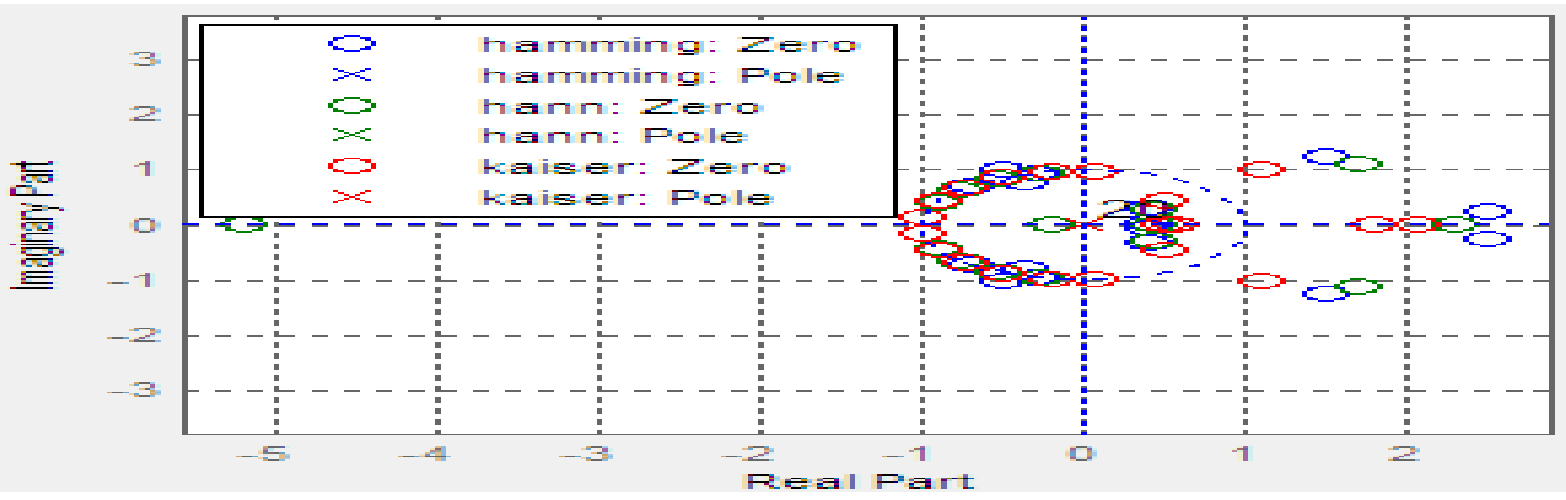

Poles and zeros plot comparison of Hamming, Hanning and Kaiser

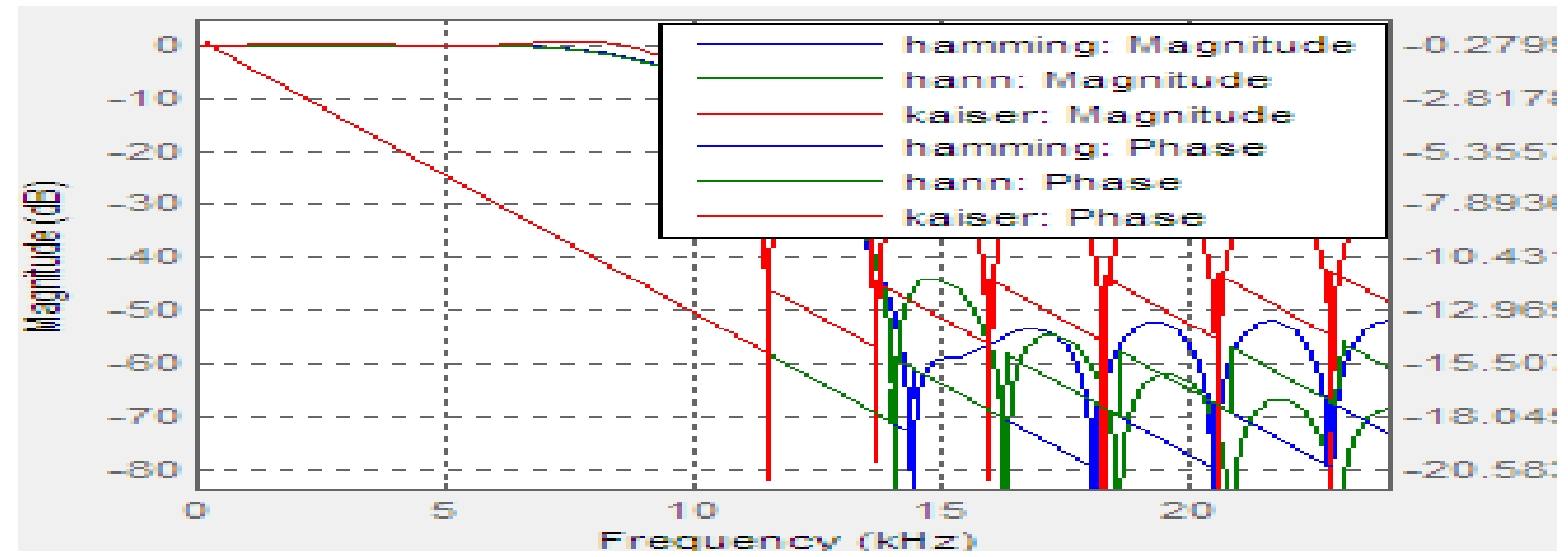

Magnitude and phase comparison of Hamming, Hanning and Kaiser 


\section{RESULT}

Table(2). Simulation Result in MATLAB

\begin{tabular}{|c|l|l|l|}
\hline Window Technique & Leakage factor & Relative sidelobe attenuetion & Mainlobe width(-3dB) \\
\hline Hamming & $0.04 \%$ & $-40.4 \mathrm{~dB}$ & 0.13281 \\
\hline Hanning & $0.05 \%$ & $-31.5 \mathrm{~dB}$ & 0.14844 \\
\hline Kaiser & $8.42 \%$ & $-13.6 \mathrm{~dB}$ & 0.65938 \\
\hline
\end{tabular}

\section{CONCLUSION}

In this paper proposed FIR low pass filter has been designed and simulated hamming, hanning and Kaiser. In hamming and hanning window technical design shows more unwanted attenuation in stop band region whereas in Kaiser window design shows less equi repel in stop band region shows from the above, it is concluded that Kaiser window design provides better result as compare to other.

\section{REFERENCES}

[1] Er. Sandeep Kaur, Er. Sangeet Pal Kaur Design of FIR fillter using hanning window, hamming window and modified hamming window. Volume 4 Issue 5, May 20152440 ISSN: 2278 - 1323 All Rights Reserved @ 2015 IJARCET

[2] Rahul Kurrey, Varsha Nirala Optimal Design of Low Pass Filter by Parzen, Taylor and Hmming Technique. Volume 5 Issue 2, February 2017 ISSN: $2321-9653$

[3] Sonika Agarwal, Aashish Gagneja, Aman Panghal Design of FIR Filter Using GA and its Comparison with Hamming window and Parks McClellan Optimization Techniques. Volume 2, Issue 7, July 2012 ISSN: 2277 128X

[4] John G. Prokis, Dimitris G.Manolaki “Digital Signal Processing” Fourth Edition. 2007.

[5] Pramod Yadav, Shankarlal Sahu, Vijendra Prajapati, Design a low pass fir filter using rectangular and taylor window. Vol. 3 Issue 8, August 2016 ISSN (Online) 2348 - 7968 | Impact Factor (2015) - 4.332

[6] Fatima, W. "Design and Analysis of Non recursive Digital Filters (FIR)", thesis, University of technology, 1999

[7] Carrara, W.G., R.M. Majewski and R.S. Goodman, "Spotlight Synthetic Aperature Radar Signal Processing Algorithms", Artech Hou se Publishers, Boston, Appendix D.2, 1995

[8] Sweta Manikpuri, Akanksha Mahto, Muskan Chaubey. Design and performance Analysis of FIR filter using hamming and hanning window Technique,VOLUME-3,ISSUE-8,AUG-2016

\section{BIOGRAPHIES}

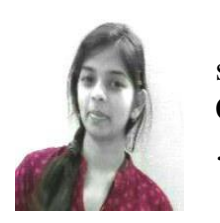

Akanksha Mahto pursuing Bachelor of Engineering in Electrical and Electronics Engineering in 6th semester from Institute of Technology Korba, Chhattisgarh Swami Vivekananda Technical University, Chhattisgarh, India

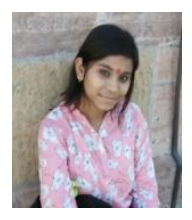

Muskan Chaubey puruing Bachelor of Engineering in Electrical and Electronics Engineering in 6th semester from Institute of Technology Korba, Chhattisgarh Swami Vivekananda Technical University, Chhattisgarh, India.

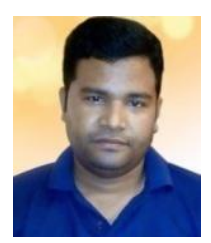

Pranay Kumar Rahi received the Bachelors of Engineering degree in Electronics and Telecommunication Engineering from Government Engineering College, Guru Ghasidas University, Bilaspur, Chhattisgarh, India in 2004,working as a Assistant Professor in Electrical and Electronic Engineering of Institute of Technology Korba since 2008 and pursuing Masters of Engineering in Electronics and Communication Engineering from National Institute of Technical Teacher's Training\& Research, Punjab University, Chandigarh, India. Presently working as assistant professor in Department of Electrical and Electronic Engineering, Institute of Technology Korba,Chhattishgarh since 2008. He has authored More than 40 research publications and published journal papers and research papers in the leading International and National journal. His primary research interest includes Digital Signal Processing, VLSI Design, Control Systems and Digital Electronics and logic design 